\title{
Identification of Local Citrus Breeds Using the Start-Codon Targeted Polymorphism Technique Combined with Clonal Sequencing
}

\author{
Yanjun Guo, Qianhua Ji , Liying Guo, Yapin Hu, Hui Jiang and Xiqin Zhou \\ Fruit Tree Research Institute, Zhaoqing University, Guangdong 526061, China \\ "For correspondence: qhgee@163.com \\ Received 29 July 2020; Accepted 03 September 2020; Published 10 December 2020
}

\begin{abstract}
This study explored the genetic diversity of 35 citrus accessions using start-codon targeted (SCoT) markers. Total 15 primers were used to amplify products ranging in length from 100 to $2000 \mathrm{bp}$. A total of 133 fragments were amplified and found that $126(95 \%)$ were polymorphic. The genetic similarity coefficients among the 35 accessions ranged from 0.67 to 1.00 , indicating that the SCoT markers could reveal high genetic diversity in the citrus germplasm. A cluster analysis showed that local citrus breeds of Sihui city fell into one cluster, possibly reflecting the geographical distribution of the tested samples. The sizes of the fragments of eight local citrus cultivars amplified by one of the primers ranged from 951 to $1001 \mathrm{bp}$, and the similitude was $95.17 \%$ high. Both single-base mutations, insertions and deletions were identified among the fragments and comparison with a sequence database suggested that the amplified region was part of a ribosomal protein-coding sequence. Using Scot marks combined with clonal sequencing, each of the test samples had one or more mutation sites that could be used as markers to differentiate from the other seven test samples. Thus, the resolution of the genetic diversity among the local citrus breeds revealed by the SCoT technique was enhanced by the subsequent sequencing analysis of specific fragments. The experimental results also provide evidence that the relationship between Citrus nobilis Lour. 'gonggan' and C. haniana Hort. ex Tseng 'Sihuihanggan' is that between parent and offspring hybrids and not between bud-mutation strains. The SCoT marker is a targeted gene molecular marker, based on the characteristic bands of primers, it can be used as a marker to isolate genes of local citrus varieties and also for investigating mutational hotspots of these gene. (C) 2021 Friends Science Publishers
\end{abstract}

Keywords: Citrus; SCoT; Local germplasm resources; Sequence alignment

\section{Introduction}

Start-codon targeted (SCoT) polymorphism is a technique for developing functional DNA markers (Collard and Mackill 2009; Xiong et al. 2009) by amplifying the region flanking the translation-initiation site (ATG) of a target gene by using a single primer. The SCoT technique was first validated in rice and features simple operation, easy establishment of technical systems for various species, effective production of markers linked with phenotypic characters, and facilitation of molecular marker-assisted breeding. SCoT primers generate reproducible results, are easy to design for a variety of species, and can be used for diversity analysis in plants, QTL mapping, bulked segregation analysis, and other applications. The SCoT technique has been used to generate molecular markers for target genes in rice (Collard and Mackill 2009), peanut (Xiong et al. 2010), mango (Luo et al. 2011), longan (Chen et al. 2010a, b), citrus (Han et al. 2011; Jiang et al. 2011), and other plants. These markers are especially useful for citrus plants because of the many new local breeds and breeds with mutated genes that have been generated through natural hybridization, artificial cultivation, selection, and cultivation based on the likeliness of hybridization, open pollination, and asexual reproduction, all of which complicate its classification.

Sihui city in the Zhaoqing region of Guangdong province has abundant citrus resources and a long history of citrus cultivation that can be traced back to the Han dynasty. The citrus breeds currently planted in the Zhaoqing region are mainly historical farm varieties with unknown origins, and there is a lack of systematic research and breeding experiments. The randomness of breed cultivation in different periods has resulted in the loss of citrus genetic resources in the region, especially for some farm varieties. In a previous study on citrus germplasm accessions of Sihui city, we studied the classification status of the local citrus breeds by field cultivation, isozyme analysis, quality analysis, and random amplified polymorphic DNA (RAPD) and simple sequence repeat (SSR) marker-based analyses of the parental species of existing natural hybrid cultivars based on chloroplast genes (Ji and Guo 2011; Ji et al. 2007, 2010, 2011a, b, 2012, 2013; Guo et al. 2013). 
In the present study, 35 citrus samples to determine the genetic diversity and genetic relationships using SCoT analysis among the local citrus breeds of Sihui city were collected. This study also sequenced target fragments from different clones of local varieties to identify its genetic variation, which can provide evidence of breeding patterns.

\section{Materials and Methods}

\section{Germplasm samples}

The 35 germplasm samples (Table 1) included Citrus (33 samples), Poncirus (one sample) and Atalantia (one sample, used as an outgroup) species collected from the Fruit Tree Research Institute of Zhaoqing University, the Guangdong Province Academy of Agricultural Sciences, and the Fruit Tree Research Institute of Chaozhou city. The represented samples were existing cultivated breeds and accessions of Sinocitrus or related plant species collected in China, including tangerines, oranges, pomelos, lemons, and other fruits. Those samples are representative to some extent and can be applied in molecular marker research.

The citrus breeds historically cultivated in Sihui city include Citrus haniana Hort. ex Tseng 'Sihuihanggan', $C$. flamea Hort. ex Tseng shiyueju 'Big fruit shape', $C$. flamea Hort. ex Tseng 'bayueju', $C$. flamea Hort. ex Tseng 'Mashiju', C. sinensis osbeck 'Lanhuacheng No.4', C. flamea Hort. ex Tseng 'wuyueju', $C$. nobilis Lour. 'gonggan', and $C$. haniana Hort. ex Tseng 'Sihuigan'. $C$. flamea Hort. ex Tseng shiyueju and $C$. nobilis Lour. 'gonggan' are the dominant breeds currently planted in the Zhaoqing region. The scientific names of "China Fruit notes: Citrus research" (Zhou and Ye 2009) were adopted for all the test samples.

\section{DNA extraction}

A modified CTAB (hexadecyl trimethyl ammonium bromide) method (Ji et al. 2011a) was used to extract DNA. The quality of the extracted DNA was determined by $1.5 \%$ agarose-gel electrophoresis and spectrophotometry. The samples were then diluted to the required concentration and stored at $-20^{\circ} \mathrm{C}$ for later use.

\section{Primer synthesis}

Fifteen selected primers were synthesized by Shanghai Bioengineering Co., Ltd. (Table 2) according to the methods of Collard and Mackill (2009). From the primers of a new gene-targeted marker developed in paddy rice and published by these authors, and by referring to the frequency of references by other researchers to fruits such as citrus, longan, grape, and pineapple, 15 primers were selected for this study.
Table 1: Samples tested

\begin{tabular}{|c|c|}
\hline Material & Sample name (Latin or English) \\
\hline 1 & Citrus grandis (L.) Osbeck'kekouyou’ \\
\hline 2 & C. tangerina Tanaka 'jiangxihongju' \\
\hline 3 & C. haniana Hort. ex Tseng 'sichuan suanju' \\
\hline 4 & C. haniana Hort. ex Tseng 'yanshanhongpi suanju' \\
\hline 5 & C. limon (L.) Burm.f.'Eureka Lemon’ \\
\hline 6 & C. sinensis Osbeck 'Newhall Navel Orange' \\
\hline 7 & C. tangerina Gongneiyiyuan'Miyauchi Iyokan' \\
\hline 8 & C. reticulata Blanco'xinshengxi NO. 3 Ponkan' \\
\hline 9 & C. reticulata $\times$ C. sinensis 'Moketeju (W.Murcott) tangor' \\
\hline 10 & C. unshiu Marcow. Nangan No.20' \\
\hline 11 & Qiuhuijuyou 'Fallglo Tangelo' \\
\hline 12 & C. reticulata Blanco 'No. 830 ponkan' \\
\hline 13 & Nowajuyou 'Nova tangelo' \\
\hline 14 & C. sinensis Osbeck 'Seike Navel orange' \\
\hline 15 & Atalantia buxifolia (Poir.) Oliv. \\
\hline 16 & Fortunella Swing \\
\hline 17 & C. haniana Hort. ex Tseng 'deqing suanju' \\
\hline 18 & C. limonia Osbeck 'Honglimeng' \\
\hline 19 & C. sinensis Osbeck 'Valencia Orange' \\
\hline 20 & C. nobilis Lour. Xingjinwenzhoumigan'okitsu wase' \\
\hline 21 & Poncirus trifoliata (L.) Raf. 'changyangzhicheng' \\
\hline 22 & C. haniana Hort. ex Tseng 'Sihuihanggan' \\
\hline 23 & C. chuana Hort. ex Tseng 'Nanfengmiju' \\
\hline 24 & C. sinensis osbeck 'tangcheng' \\
\hline 25 & C. sinensis osbeck 'hongjiangcheng' \\
\hline 26 & C. flamea Hort. ex Tseng shiyueju'Big fruit shape' \\
\hline 27 & C. grandis (L.) osbeck 'shatianyou' \\
\hline 28 & C. flamea Hort. ex Tseng ‘bayueju’ \\
\hline 29 & C. flamea Hort. ex Tseng 'Mashiju' \\
\hline 30 & C. sinensis osbeck 'Lanhuacheng No.4' \\
\hline 31 & C. junons Sieb. ex Tanaka \\
\hline 32 & C. flamea Hort. ex Tseng wuyueju \\
\hline 33 & C. nobilis Lour. 'gonggan' \\
\hline 34 & C. haniana Hort. ex Tseng 'Sihuigan' \\
\hline 35 & Poncirus trifoliata (L.) Raf \\
\hline
\end{tabular}

Table 2: SCoT primer used

\begin{tabular}{ll}
\hline SCoT primer & Primer sequence \\
\hline S1 & CAACAATGGCTACCACCA \\
S3 & CAACAATGGCTACCACCG \\
S8 & CAACAATGGCTACCACGT \\
S9 & CAACAATGGCTACCAGCA \\
S11 & AAGCAATGGCTACCACCA \\
S12 & ACGACATGGCGACCAACG \\
S17 & ACCATGGCTACCACCGAG \\
S19 & ACCATGGCTACCACCGGC \\
S22 & AACCATGGCTACCACCAC \\
S23 & CACCATGGCTACCACCAG \\
S24 & CACCATGGCTACCACCAT \\
S25 & ACCATGGCTACCACCGGG \\
S26 & ACCATGGCTACCACCGTC \\
S34 & ACCATGGCTACCACCGCA \\
S36 & GCAACAATGGCTACCACC \\
\hline
\end{tabular}

\section{SCoT fragment amplification}

SCoT fragments were amplified in a $20 \mu \mathrm{L}$ optimized PCR system as described by Jiang et al. (2011). The reaction system contained $80 \mathrm{ng}$ DNA template, $1.6 \mathrm{~m} M \mathrm{Mg}^{2+}, 0.3$ $\mathrm{m} M$ dNTPs, $0.2 \mu M$ primers, and $1.6 \mathrm{U}$ TaqDNA polymerase. The reaction conditions were as follows: predenaturation at $94^{\circ} \mathrm{C}$ for $4 \mathrm{~min} ; 35$ cycles of denaturation at $94^{\circ} \mathrm{C}$ for $1 \mathrm{~min}$, annealing at $50^{\circ} \mathrm{C}$ for $45 \mathrm{~s}$, and extension at 
$72^{\circ} \mathrm{C}$ for $1 \mathrm{~min}$; and extension at $72^{\circ} \mathrm{C}$ for $8 \mathrm{~min}$ after the cycling. The PCR products were stored at $4{ }^{\circ} \mathrm{C}$ and later verified by $1.5 \%$ agarose-gel electrophoresis.

\section{SCoT fragment amplification, cloning, and sequencing}

Eight samples for SCoT fragment amplification, cloning, and sequencing were all from local citrus breeds of Sihui city [(1) C. haniana Hort. ex Tseng 'Sihuihanggan', (2) $C$. flamea Hort. ex Tseng shiyueju 'Big fruit shape', (3) $C$. flamea Hort. ex Tseng 'bayueju', (4) C. flamea Hort. ex Tseng 'Mashiju', (5) C. sinensis osbeck 'Lanhuacheng No.4', (6) C. flamea Hort. ex Tseng 'wuyueju', (7) $C$. nobilis Lour. 'gonggan', and (8) C. haniana Hort. ex Tseng 'Sihuigan'].

There were two reasons for selecting primer S19 for amplification, cloning and sequencing of SCoT fragments. Reference 8 applied this primer to the bud mutation identification of citrus varieties with close genetic relationships, and it was speculated that the eight local varieties may be closely related. If S19 amplified a common characteristic band, it was proposed to try to discover some rules from its sequence.

The amplification products of the eight samples amplified by SCoT primer S19 were verified by $1.5 \%$ agarose gel electrophoresis. The T1Angel Midi Purification Kit (T1ANGEN) was used to recycle and purify the characterized bands for TA cloning, then for connection using the pMD19T cloning vector (Takara Biotechnology (Dalian) Co., Ltd., China) as the carrier, and the connection products were transformed into competent TOP10 cells. Positive clones were identified by dye PCR amplification of templates from single colonies and sent to Shanghai Bioengineering Co., Ltd. for sequencing.

\section{Sequence analysis}

Chromas1.45 was used to view the traces of the sequencing results. Artificial proofreading and editing were used to ensure that the sequences were correct, and then the ContigExpress software was used for sequence splicing. After the sequences were determined, Clustalx 1.81 was used for comparative analysis of multiple sequences (http: //www.hgmp.mrc.ac.uk/Registered/Option/clustalx.html), and BLAST was used to identify comparative similarities (National Center for Biotechnology Information of USA, NCBI website: http://www.ncbi.nlm.nih.gov/).

\section{Results}

\section{Primer amplification}

All 15 primers generated clear polymorphic bands (Fig. 1 ). The number of amplified bands ranged from 1 to 11 . A total of 133 bands were amplified, of which 126 were polymorphic. With each primer producing eight polymorphic bands on average, the average polymorphism rate was $95 \%$.

1 and 0 data regarding all bands were clustered analysis by Hierarchical clustering method, and then dendrogram was constructed (Fig. 2). Using a genetic similarity coefficient of 0.73 as the criterion, the test samples were divided into three groups. Group 1 consisted of Atalantia buxifolia (Poir.) Oliv, group 2 Fortunella Swing, Poncirus trifoliata (L.) Raf, and P. trifoliata (L.) Raf. 'Changyangzhicheng' and group 3 mandarin, tangerine, orange, lemon, pomelo, and other varieties. Using a genetic similarity coefficient of 0.81 as the criterion, the lemon, pomelo, and mandarin, tangerine, and orange in group 3 could be divided into different subgroups. In this group 3 , the local citrus breeds of the Zhaoqing region formed a single cluster, indicating a high degree of genetic relatedness. The similarity coefficients between $C$. nobilis Lour. 'Gonggan' and $C$. haniana Hort. ex Tseng 'Sihuihanggan', C. flamea Hort. ex Tseng shiyueju and C. flamea Hort. ex Tseng wuyueju, and C. flamea Hort. ex Tseng 'bayueju' and $C$. chuana Hort. ex Tseng 'Nanfengmiju' were $0.91,0.97$, and 0.97 , respectively; and those between $C$. haniana Hort.ex Tseng 'Sihuigan', C. flamea Hort. ex Tseng 'Mashiju', C. haniana Hort. ex Tseng 'sichuan suanju', C. haniana Hort. ex Tseng 'yanshanhongpi suanju' and $C$. haniana Hort. ex Tseng 'deqing suanju', were all 1.0.

\section{SCoT fragment sequencing}

The results show that specific primers can amplify characteristic fragments of some citrus plants and can be used in further studies. The primer S19 was selected, as it amplifies a common, obvious characteristic band in eight Sihui local citrus varieties, and it was also applied in reference 8 to identify bud mutation in citrus plants. The selection of S19 was appropriate for the present study and for comparative analysis in other studies.

The DNA fragments from the local citrus breeds of Sihui city [(1) C. haniana Hort. ex Tseng 'Sihuihanggan', (2) C. flamea Hort. ex Tseng shiyueju 'Big fruit shape', (3) C. flamea Hort. ex Tseng 'bayueju', (4) C. flamea Hort. ex Tseng 'Mashiju', (5) C. sinensis osbeck 'Lanhuacheng No.4', (6) C. flamea Hort. ex Tseng 'wuyueju', (7) $C$. nobilis Lour. 'gonggan', and (8) C. haniana Hort. ex Tseng 'Sihuigan'] amplified by primer S19 were recycled for TA cloning and sequencing. The total lengths of the amplified fragments ranged from 951 to $1001 \mathrm{bp}$. Alignment analysis revealed that the SCoT sequences of the eight local breeds were highly similar, with identity as high as $95.17 \%$. There were a total of 39 mutation sites, including $\mathrm{A}>\mathrm{G}, \mathrm{T}>\mathrm{C}$, and $\mathrm{C}>\mathrm{T}$ conversions as well as $\mathrm{T}>\mathrm{G}, \mathrm{G}>\mathrm{T}$, and $\mathrm{A}>\mathrm{T}$ transversions, a $(\mathrm{G})$ deletion, and insertions at different sites (Table 3 ).

The BLAST results showed that the second halves (starting between nucleotides 720 and 950) of the amplified 


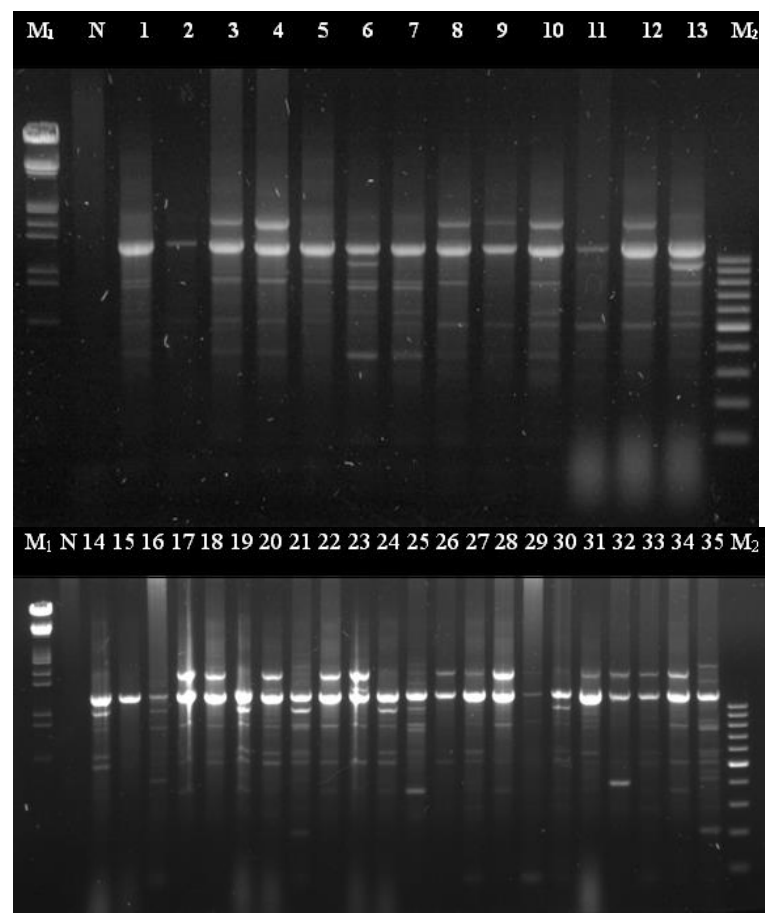

Fig. 1: Amplification of 35 citrus accessions by the $\mathrm{S} 1$ primer

Note: M1: $\lambda$ DNA/EcoR I + Hind III; M2: 100-bp marker; N: blank control. The accessions are numbered 1-35 (Table 1); the S1 primer amplified eight polymorphic fragments among them

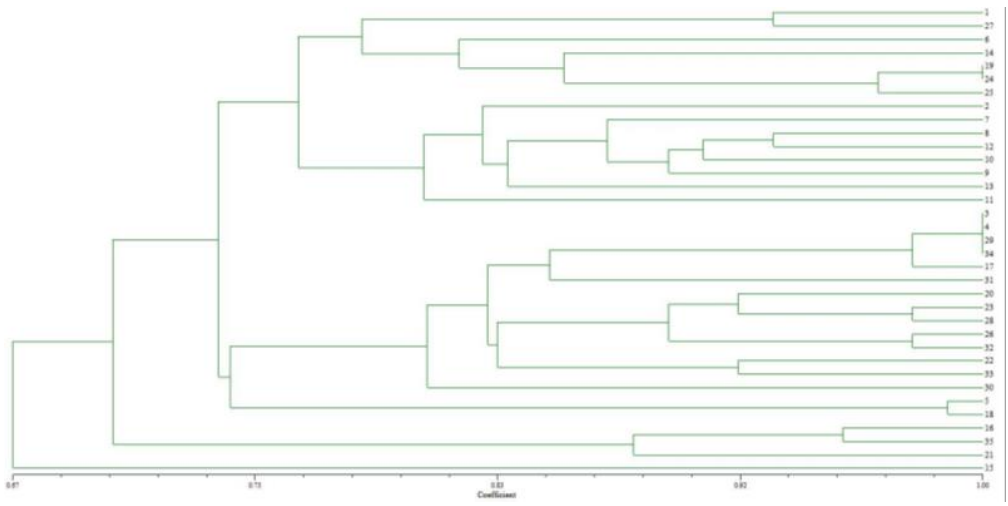

Fig. 2: Dendrogram of 35 citrus accessions based on SCoT fragments Note: The accessions are numbered 1-35 (Table 1)

fragments of $C$. haniana Hort. ex Tseng 'Sihuihanggan', $C$. flamea Hort. ex Tseng 'bayueju', C. sinensis osbeck 'Lanhuacheng No.4', C. flamea Hort. ex Tseng 'wuyueju', and $C$. haniana Hort. ex Tseng 'Sihuigan' were of the same origin and presented in a positive permutation. The first halves (starting between nucleotides 1 and 370) of the amplified fragments of $C$. flamea Hort. ex Tseng shiyueju 'Big fruit shape', C. flamea Hort. ex Tseng 'Mashiju', and C. nobilis Lour. 'gonggan' were of the same origin and presented in a reversed permutation. Comparative analysis and alignment following the reverse complementation of sequences of $C$. flamea Hort. ex Tseng shiyueju 'Big fruit shape', C. flamea Hort. ex Tseng 'Mashiju', and C. nobilis Lour. 'gonggan' revealed high similarity (Fig. 3).
The similar sequence in the fragments from the eight samples had the same origin as the mitochondrial 60 s ribosomal protein gene sequence on rice chromosome 12 , with $90 \%$ identity with the sequences in this study. It also had the same origin as the gene for the mitochondrial S3 ribosomal protein of beet, with 90\% identity with the sequences, origin was also similar as genes in the mitochondrial genomes of apple, tobacco, and petunia. These results suggest that the SCoT primers amplified part of a gene encoding a ribosomal protein that may originate in the mitochondrial genome. However, these results do not exclude the possibility that the amplified fragment originated in the nuclear genome. 


\section{Discussion}

Samples of three citrus-related genera (Citrus, Poncirus, and Atalantia) were selected in this study. The clustering results showed that the SCoT technique revealed genetic diversity among the tested samples, clustering samples of different genera separately, a result largely consistent with previous studies using RAPD and SSR markers (Ji et al. 2011c, 2012). In this study, as the hybrid (P. trifoliata $\times C$. sinensis) of Fructus aurantii and sweet orange, citrange (Poncirus trifoliata (L.) Raf. Changyangzhicheng), is categorized as citrus using RAPD and SSR markers, and its SCoT clustering result is the same as for Fructus aurantii, suggesting homology with trifoliate orange.

The citrus breeds of Sihui city clustered into one category when the genetic coefficient was 0.80 , and the genetic relationship was closer, indicating that geographical factors strongly influence the study of genetic relationships among citrus. In ancient China, the exchange of citrus plants among different areas was relatively rare; therefore, citrus varieties display typical geographical distributions. For citrus plants, distant hybridization by intergeneric and interspecific crossing is easy to achieve and has generated many distant natural hybrids in nature. The circulation of citrus varieties in different regions in modern times and the rapid promotion of citrus breeding are beneficial to citrus germplasm diversity (Deng and Peng 2013). Some local varieties in Sihui are not large-scale cultivated varieties with small external circulation, and most of them are farm varieties, hybrids or natural bud mutations that were not intentionally developed; for this reason the SCoT clustering result still shows common geographical distributions.

SCoT molecular markers have been successfully applied to plant genetic diversity, genetic relationship identification and genetic map construction (Zhai et al. 2018). It has been pointed out that the SCoT marker was suitable for interspecific genetic analysis and identification of distant hybrid progenies, but unsuitable for genetic analysis of different types of common tobacco species (Liu et al. 2013). Through SCoT study, there was no significant difference among the bud-mutation varieties of sweet orange, and the same bands were amplified by single primer in some bud-mutation varieties (Jiang et al. 2011). In this study, primer S19 amplified the same specific band in all the citrus breeds of Sihui city, suggesting a region of homology among the test samples. However, recycling the bands amplified by the S19 primer for TA cloning and sequencing showed that all the samples had a very high degree of sequence identity and some differences. Each of the test samples had one or more mutation sites that could be used as markers to differentiate from the other seven test samples. The similar genetic backgrounds among the citrus breeds of Sihui city could result from the communication of partial genetic materials via natural hybridization or the formation of different breeds and strains via bud mutations. However, the sequencing results showed that over years of cultivation,
Table 3: Variation among the S19 sequences of eight Citrus species

\begin{tabular}{|c|c|c|c|c|c|c|c|c|}
\hline \multirow[t]{2}{*}{ No. } & \multicolumn{8}{|c|}{ material } \\
\hline & $\overline{1}$ & 2 & 3 & 4 & 5 & 6 & 7 & 8 \\
\hline 167 & - & - & - & - & - & - & $\mathrm{T}$ & - \\
\hline 171 & - & - & - & - & - & - & A & - \\
\hline 183 & - & - & - & - & - & - & G & - \\
\hline 190 & - & - & - & - & - & - & A & - \\
\hline 199 & $\mathrm{~T}$ & $\mathrm{~T}$ & $\mathrm{~T}$ & $\mathrm{~T}$ & $\mathrm{~T}$ & $\mathrm{~T}$ & G & $\mathrm{T}$ \\
\hline 205 & - & - & - & - & - & - & $\mathrm{C}$ & - \\
\hline 214 & G & G & G & G & $\mathrm{G}$ & G & $\mathrm{T}$ & G \\
\hline 216 & - & - & - & - & - & - & A & - \\
\hline 225 & - & - & - & - & - & - & $\mathrm{C}$ & - \\
\hline 227 & G & G & G & G & G & G & $\mathrm{T}$ & G \\
\hline 232 & $\mathrm{C}$ & $\mathrm{T}$ & $\mathrm{T}$ & $\mathrm{T}$ & $\mathrm{T}$ & $\mathrm{T}$ & $\mathrm{T}$ & $\mathrm{T}$ \\
\hline 237 & - & - & - & - & - & - & A & - \\
\hline 239 & $\mathrm{C}$ & $\mathrm{C}$ & $\mathrm{C}$ & $\mathrm{C}$ & $\mathrm{C}$ & $\mathrm{C}$ & $\mathrm{T}$ & $\mathrm{C}$ \\
\hline 247 & - & - & - & - & - & - & A & - \\
\hline 261 & - & - & - & - & - & - & $\mathrm{C}$ & - \\
\hline 270 & - & - & - & - & - & - & $\mathrm{C}$ & - \\
\hline 321 & A & G & A & A & A & A & A & A \\
\hline 374 & A & A & A & A & A & $\mathrm{T}$ & A & A \\
\hline 447 & C & $\mathrm{T}$ & $\mathrm{T}$ & $\mathrm{T}$ & $\mathrm{T}$ & $\mathrm{T}$ & $\mathrm{T}$ & $\mathrm{T}$ \\
\hline 468 & G & - & G & G & $\mathrm{G}$ & G & G & G \\
\hline 556 & $\mathrm{~T}$ & $\mathrm{~T}$ & $\mathrm{~T}$ & $\mathrm{~T}$ & $\mathrm{~T}$ & $\mathrm{~T}$ & $\mathrm{C}$ & $\mathrm{T}$ \\
\hline 571 & A & G & A & A & A & A & A & A \\
\hline 604 & G & A & A & A & A & A & A & A \\
\hline 625 & $\mathrm{~T}$ & $\mathrm{~T}$ & $\mathrm{~T}$ & $\mathrm{~T}$ & $\mathrm{~T}$ & $\mathrm{C}$ & $\mathrm{T}$ & $\mathrm{C}$ \\
\hline 675 & $\mathrm{~T}$ & $\mathrm{~T}$ & $\mathrm{~T}$ & $\mathrm{~T}$ & $\mathrm{~T}$ & $\mathrm{~T}$ & $\mathrm{C}$ & $\mathrm{T}$ \\
\hline 718 & A & A & A & G & A & A & A & A \\
\hline 792 & A & A & A & A & A & A & $\mathrm{T}$ & A \\
\hline 893-895 & TTA & TTA & --- & TTA & --- & TTA & TTA & TTA \\
\hline $897-899$ & --- & --- & --- & --- & --- & --- & TAT & --- \\
\hline 913 & A & A & A & A & A & A & A & G \\
\hline 928 & - & - & - & - & - & - & - & $\mathrm{C}$ \\
\hline 945 & - & - & - & - & - & - & - & G \\
\hline 947 & $\mathrm{~T}$ & $\mathrm{~T}$ & $\mathrm{~T}$ & $\mathrm{~T}$ & $\mathrm{~T}$ & $\mathrm{~T}$ & $\mathrm{~T}$ & $\mathrm{~T}$ \\
\hline 961 & - & - & - & - & - & - & - & C \\
\hline te: Nun & 18 & he test & & $\operatorname{see} \mathrm{N}$ & ials & $\mathrm{d} \mathrm{m}$ & $\mathrm{s}$ section & \\
\hline
\end{tabular}

Fig. 3: Dendrogram of eight local Citrus species based on sequencing of S19 fragments

Note: The species are numbered 1-8; see Materials and Methods

differences have appeared among the breeds owing to internal mutations in the same functional gene. Thus, the resolution of the genetic diversity among the local citrus breeds revealed by the SCoT technique was enhanced by the subsequent sequencing analysis of specific fragments.

C. nobilis Lour. 'gonggan', one of the dominant citrus breeds planted in the Zhaoqing region, is thought to be a natural hybrid of tangerine and orange. Previously, using RAPD and SSR markers it was shown that $C$. nobilis Lour. 'gonggan' and $C$. haniana Hort. ex Tseng 'Sihuihanggan' have the closest genetic relationship among the local breeds (Ji et al. 2012, 2013), consistent with the results of the present study. Some researchers speculate that $C$. haniana Hort. ex Tseng 'Sihuihanggan' is the female parent of $C$. nobilis Lour. 'gonggan' according to evidence based on the 
trnl chloroplast gene (Ji et al. 2010). Therefore, C. nobilis Lour. 'gonggan' is probably a hybrid of $C$. haniana Hort. ex Tseng 'Sihuihanggan' and orange. The possibility that it is instead a bud-mutation strain of $C$. haniana Hort. ex Tseng 'Sihuihanggan' is not excluded, although there is currently no evidence supporting this hypothesis. The sequencing results showed that the SCoT sequence amplified by primer S19 contains 20 single-base polymorphism and a three-base polymorphism between $C$. nobilis Lour. 'gonggan' and $C$. haniana Hort. ex Tseng 'Sihuihanggan'. Sixteen of the single-base mutations were concentrated between nucleotide positions 160 and 270, indicating a high mutation frequency in the region. Similar results are displayed between $C$. nobilis Lour. 'gonggan' and several other samples. The base sequence in this region forms mutational hotspots in this gene of this variety and is mainly dominated by insertion mutation, followed by transversion and then transition mutation. The cause of mutational hotspots of citrus is still unclear. Theoretically, there is a higher frequency of transition in mutational hotspots and it is much easier for insertion and deletion mutation to occur in short tandemly duplicated sequence. Given that the SCoT marker is a targeted gene molecular marker, the characteristic bands of primers can be used as a marker to isolate genes of local citrus varieties in Sihui city and for investigating mutational hotspots of this gene. It can also be used to recognize and analyze potential mutational hotspots.

The SCoT study of Jiang et al. (2011) on bud-mutation strains of sweet orange showed that their genetic backgrounds are very narrow and differences between the aligned sequences amplified by a single SCoT primer can be minimal. There were very few differences between some breeds, indicating that the mutation range of the budmutation strains is narrow.

In the present study, the SCoT genetic similarity coefficient of $C$. nobilis Lour. 'gonggan' and $C$. haniana Hort. ex Tseng 'Sihuihanggan' was high, indicating a close genetic relationship. There were obvious differences among the fragments amplified by some SCoT primers, and the sequencing of the same characteristic sequence amplified by the single primer S19 showed multiple mutation sites, suggesting that the relationship between $C$. nobilis Lour. 'gonggan' and $C$. haniana Hort. ex Tseng 'Sihuihanggan' is between parent and offspring hybrids and not between budmutation strains.

Thus, it is speculated that based on the homologoussequence alignment results, the fragment amplified by primer S19 is part of a ribosomal protein-coding sequence, which may originate in the mitochondrial or nuclear genome. The evidence based on the chloroplast trnl genes of C. nobilis Lour. 'gonggan' and C. haniana Hort. ex Tseng 'Sihuihanggan' suggests that $C$. haniana Hort. ex Tseng 'Sihuihanggan' is the female parent of $C$. nobilis Lour. 'gonggan'. Based on the SCoT sequencing results, however, the $C$. haniana Hort. ex Tseng 'Sihuihanggan' sequence had a higher mutational frequency than the $C$. nobilis Lour. 'gonggan' sequence, indicating that the sequence encoding ribosomal protein is more likely from the nuclear than from the mitochondrial genome and is probably from the male parent of $C$. nobilis Lour. 'gonggan'. The ribosomal protein may participate in ribosome structure and translation, which plays an important role in plant development. Its protein synthesis starts from the $\mathrm{N}$ terminus and its sequence composition has a large effect on its biological function. Study of the protein and its $\mathrm{N}$ end sequence is beneficial for the analysis of protein structure and the revelation of the biological function of the protein. Base mutations in the tested accessions may account for amino acid variation at the $\mathrm{N}$ end, a subject that awaits further research.

Several types of target-gene molecular markers have been developed and used in recent years. As a new method, SCoT markers are an effective supplement to RAPD, ISSR, and other conventional random DNA markers (Collard and Mackill 2009), which can produce markers linked with phenotypic characters to facilitate molecular marker-assisted breeding. Given that only 15 SCoT primers were studied, and detection analysis was performed only on one primer combining some single genes, the information obtained is limited. In future research, further development of other polymorphic SCoT primers should be performed, combining sequencing results from fragments amplified by multiple SCoT primers, for the study of local citrus germplasm resources in Sihui city. Several types of targetgene molecular markers have been developed and used in recent years. As a new method, SCoT markers are an effective supplement to RAPD, ISSR, and other conventional random DNA markers (Collard and Mackill 2009), which can produce markers linked with phenotypic characters to facilitate molecular marker-assisted breeding. Therefore, it will play a significant role in the development of excellent genes of local citrus varieties.

\section{Conclusion}

The SCoT markers could reveal high genetic diversity in the citrus germplasm and could be used to study the regional distribution of citrus. Each of the test samples had one or more mutation sites that could be used as markers to differentiate from the other seven test samples, thus, the resolution of the genetic diversity and research on gene hotspots among the local citrus breeds revealed by the SCoT technique was enhanced by the subsequent sequencing analysis of specific fragments. The relationship between $C$. nobilis Lour. 'gonggan' and $C$. haniana Hort. ex Tseng 'Sihuihanggan' is that between parent and offspring hybrids and not between bud-mutation strains. The SCoT technique combined with clonal sequencing can provide a reference for the collection, protection, and effective use of the local citrus germplasm resources of the Sihui region. It can be applied to research into mechanisms, functions, and phylogenies of citrus genes and the development of target genes used as markers in citrus breeding in future. 


\section{Acknowledgements}

This study was supported by China Agriculture (Citrus) Research System Building Project (cars-26); Rural Sci-tech Commissioner Project (2019-18).

\section{Author Contributions}

YG and QJ planned the experiments, YG, LG and HJ interpreted the results, YG and $\mathrm{YH}$ made the write up and $\mathrm{YH}$ and $\mathrm{XZ}$ statistically analyzed the data and made illustrations

\section{References}

Chen H, XH He, C Luo, JH Zhu, F Li (2010a). Analysis on the genetic diversity of 24 Longan (Dimocarpus longan) accessions by SCoT markers. Acta Hortic Sin 37:1651-1654

Chen H, X He, JC Pan (2010b). Advances on application of molecular markers in Longan researches. Chin Agric Sci Bull 26:26-30

Collard BCY, DJ Mackill (2009). Start codon targeted (SCoT) polymorphism: A simple, novel DNA marker technique for generating gene-targeted markers in plants. Plant Mol Biol Rep 27:86-93

Deng XX, SA Peng (2013). Origin and classification of Citrus. In: Towards the Citrology, pp: 69-95. Zhang L (Ed.). China Agricultural Publications, Beijing, China

Guo YJ, QH Ji, XQ Zhou, YP Hu, LY Guo, H Jiang (2013). A genetic background research on Citrus nobilis Lour. 'Gonggan' based on the chloroplast trnL gene. Genet Mol Res 12:3079-3087

Han GH, SQ Xiang, WX Wang, ZG Jia, QB Hong, GL Liang (2011). Establishment and application of scot molecular marker system for citrus. Acta Hortic Sin 38:1243-1250

Ji QH, JW Zeng, YJ Guo (2013). Using optimized random amplified polymorphic DNA (RAPD) markers to identify the category status of Citrus nobilis Lour. Gonggan. Afr J Biotechnol 10:13982-13990
Ji QH, JW Zeng, YJ Guo (2012). Simple sequence repeats molecular marker analysis of the genetic relationship between Zhaoqing local fine citrus breeds and other citrus and allied plants. J Agric Sci 4:102-110

Ji QH, YJ Guo (2011). The origin of Gonggan. In: Towards the China Gonggan (Citrus nobilis Lour. 'Gonggan'), pp: 19-32. Science Press, Beijing, China

Ji QH, YJ Guo, ZF Li (2011a). Preliminary study on purity Identification for local citrus variety by using molecular method. $S$ Chin Fruits 40:6-11

Ji QH, JW Zeng, YJ Guo (2011b). Preliminary study on the origin of a good locale citrus varieties-Citrus reticulate 'gonggan'. Chin Agric Sci Bull 27:222-227

Ji QH, JW Zeng, YJ Guo (2011c). Using optimized random amplified polymorphic DNA (RAPD) markers to identify the category status of Citrus nobilis Lour. Gonggan. Afr J Biol 10:13982-13990

Ji QH, YJ Guo, JM Yao, JH He (2010). Study on leaf mineral nutrient and its effect on fruit quality of Gonggan. S W Chin J Agric Sci 23:786-790

Ji QH, YJ Guo, GJ Liang (2007). An analysis of Isoenzymes in relation to fruit quality of Gonggan. J Sichuan Agric Univ 25:425-430

Jiang QQ, GY Long, WW Li, ZN Deng (2011). Identification of genetic variation of sweet orange clones from Hunan by using scot markers and sequence analysis. Chin Agric Sci Bull 27:148-154

Liu C, JB Dang, YX Wei, TJ Wu, WX Wang, QG Guo, GL Liang (2013) The application of SCoT molecular marker technology in genetic analysis and interspecific hybrid identification in genus Nicotiana. Acta Tabac Sin 19:107-111

Luo C, XH He, H Chen, YL Wei, MJ Li (2011). Genetic diversity of mango cultivars estimated using SCoT and ISSR markers. Biochem Syst Ecol 39:676-684

Xiong FQ, J Jiang, RC Zhong, ZQ Han, LQ He, Z Li, WJ Zhuang, RH Tang (2010). Start codon targeted polymorphism for evaluation of functional genetic variation and relationships in cultivated peanut (Arachis hypogaea L.) genotypes. Mol Biol Rep 38:3487-3494

Xiong FQ, ZHL Chen, LA Pan (2009). Start codon target polymorphism (SCot): A novel gene targeted marker technique based on the translation start condon. Mol Plant Breed 7:635-638

Zhai XM, M Tang, XH Wu, J Zhang, YJ Hou (2018). Application of SCoT molecular markers in tea breeding. South Chin Agric 12:18-21

Zhou KL, YM Ye (2009). China Fruit Notes Citrus Research. China Forestry Publishing House, Beijing, PR China 